\section{El Museo de Anatomía de la Universidad de Chile, un monumento nacional}

\author{
JULIO L. CÁRDENAS V. ${ }^{1}$
}

\section{The anatomy museum of the University of Chile: a national monument}

The accrual of anatomical preparations since the nineteenth century in Santiago, Chile, became the so called "anatomical cabinet" under the supervision of professor Julio F Lafargue. Afterwards, this cabinet evolved to form an anatomical museum in the mid twentieth century. It contained preparations using corpses whose identification was not known. Now, the corpses are donated through a body donation program that started thirty years ago. The collection contains, among other interesting items, a situs inversus preparation, Juan Martel's mummy, Tramond house wax preparations and a jibarized head. Nowadays, the museum is open to the community, its collection is recognized as a national historical monument, and has links with other university museums in the country and abroad.

(Rev Med Chile 2018; 146: 1459-1465)

Key words: Anatomy; Dissection; History of Medicine; Museums.

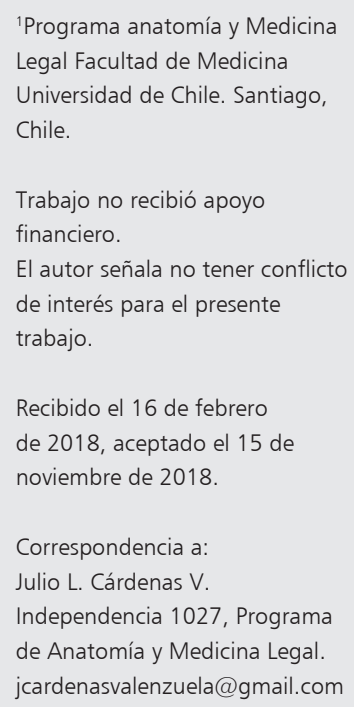

\section{Los orígenes del museo de anatomía}

I os orígenes del Museo de Anatomía de la Universidad de Chile están relacionados con la historia de la disciplina más que a la historia de la colección misma. Hasta el año de 1833 en que se inician los estudios de medicina formales en el Instituto Nacional ${ }^{1}$ ninguna alusión escrita se ha encontrado sobre la existencia de un Museo de Anatomía propiamente tal.

\section{El Gabinete de Anatomía del Dr. Lafargue}

Según Augusto Orrego $\mathrm{Luco}^{2}$, la Escuela de Medicina de la segunda mitad del s. XIX era un pequeño edificio con una gran puerta central, para pasar el carretón que llevaba los cadáveres, a cuyos lados había dos grandes ventanas con hermosas rejas. Todo el frontis era estucado y sobre su pórtico había un bajo relieve de tamaño natural que representaba una lección de Anatomía. En 1857 se había trasladado a la calle San Francisco el anfiteatro de anatomía que se componía de dos salas altas de cal y ladrillo a ambos lados; la de la izquierda con una gran estantería en un costado donde se guardaban grandes bocales de alcohol con preparaciones y objetos anatómicos destinados a la docencia; era ocupada como gabinete por el Dr. Julio Francisco Lafargue. De origen francés, brillante en sus exposiciones, hábil disector, de vastos conocimientos y notable cultura literaria. Practica las primeras inyecciones en venas y arterias efectuando sus trabajos en sus disecciones ejecutadas con asombrosa habilidad dignas de un anfiteatro europeo pero con los exiguos recursos locales. Es el iniciador de los trabajos anatómicos en Chile y por ello, el primero en establecer piezas anatómicas conservadas para luego ser utilizadas en la docencia en lo que se llamaría pomposamente museo.

En relación a las disecciones, fuente de las preparaciones anatómicas del museo, debemos recordar que desde el año 1853 se nombraba al alumno más aventajado como disector, de esta forma los primeros estudiantes dejaron de disecar 


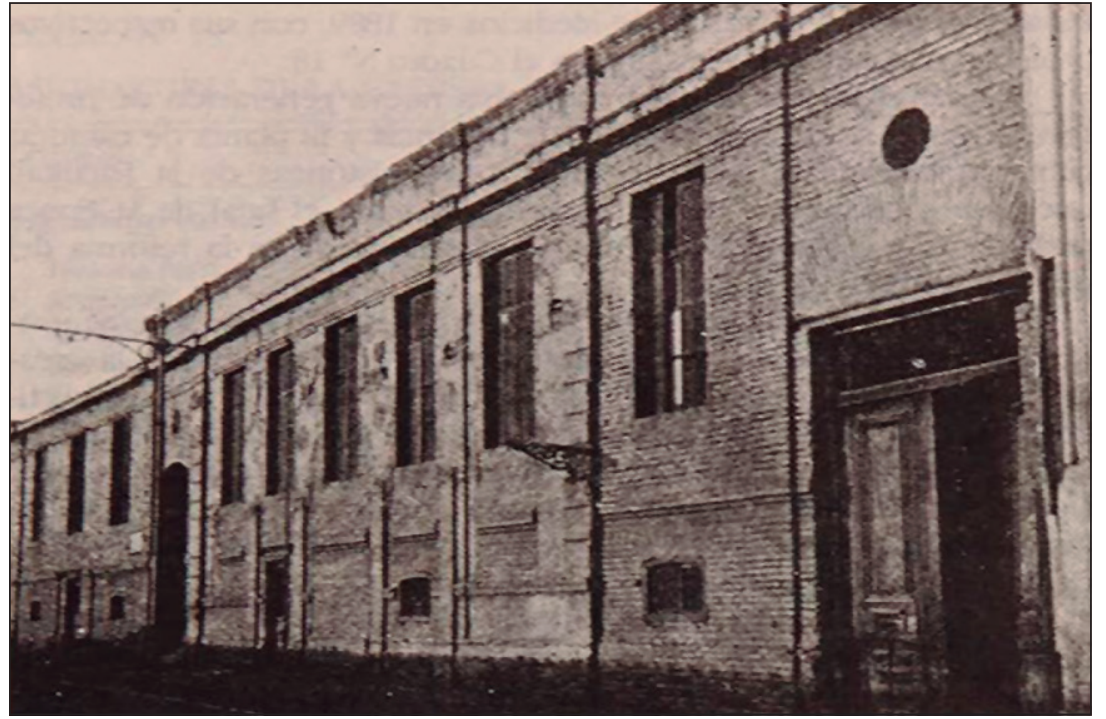

Figura 1. Escuela de Medicina, calle San Francisco. A la izquierda de la puerta de entrada, el gabinete de Lafargue. Historia Chilena de la medicina Ricardo Cruz Coke, pág. 423. como era la costumbre inicial, disminuyendo desde entonces dramáticamente la mortalidad entre los alumnos que hasta ese año se contaba en la mitad. Así, en el primer curso de Morán, de 3 alumnos murieron 2 en el tercer año; en el segundo, de 6 alumnos murieron otros 2; en el tercero, de 5 murió 1 , y en el cuarto, murió otro3. Este nombramiento dejó de ser anual desde 1860, dándole mayor importancia y jerarquía a los disectores, quienes permanecían ahora en el cargo; el disector principal recibiría posteriormente el nombre de jefe de trabajos. Se encargaría de preparar el cadáver para las demostraciones que realizaba, liberando al profesor quién se dedicaba entonces a las actividades teóricas. Desde la segunda mitad del s. XIX se acuñaría el nombre de prosector, para diferenciarlo del resto de los disectores (Figura 1).

Destaca la primera adquisición que se hace bajo la dirección de J.J. Aguirre para este gabinete de una pieza francesa que en 1865 el Gobierno adquiere en 1.000 pesos, la estatua anatómica del dr. Ansoux (sic). Era una figura en cartón piedra de tamaño natural con piezas desmontables ${ }^{4}$. Agrega el Dr. Enrique Laval: habría llegado en 1846 en forma accidental y "por ser en su género una de las obras más perfectas que pueden presentarse en Europa, según resulta del examen que han hecho de ella personas inteligentes,... "un Decreto Supremo del 26 de diciembre de ese año autoriza al director del Museo Nacional para adquirirla ${ }^{5}$ (Figura 2).

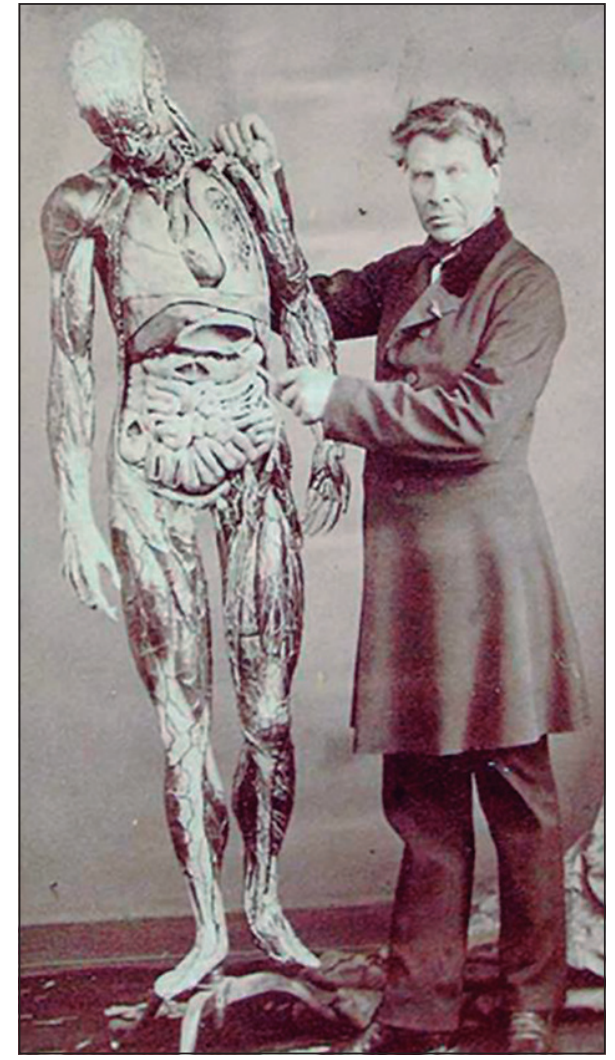

Figura 2. Estatua anatómica Dr. Auzoux. Fotografía estudio Hermanos Bisson, Francia, perteneciente al Dr. Vicente Izquierdo y donada por su familia al Museo Nacional de Medicina, Facultad de Medicina Universidad de Chile, año 2009. Gentileza Museo Nacional de Medicina. 
Después de más de 140 años, se encontraría esta estatua en el subterráneo de Morfología de la Facultad de Medicina de la Universidad de Chile, (posteriormente sabriamos que se llamaba Auzoux), Sería la pieza de uso anatómico más antigua del país ${ }^{6}$.

Le sucede a J.J. Aguirre el Dr. Pablo Zorrilla, gran disector y director del gabinete de Anatomía ubicado aún en la calle San Francisco, siendo disectores Adolfo Valderrama, Adolfo Murillo y Domingo Gutiérrez quienes contribuyen con algunos preparados para el Museo. A Zorrilla se le atribuye el proceso de la transición de gabinete, lugar para el acopio de las piezas anatómicas, a museo anatómico, lugar dispuesto de preparaciones para su exhibición. En 1867 se cuentan 176 piezas, 56 eran de anatomía patológica según señala Ferrer.

En 1873, el Dr. Ramón Allende Padín señalaría en carta dirigida a José J. Aguirre conocer algunas preparaciones de Ambrosio Coste, de origen veneciano, gran disector artista en su género y continuador de los trabajos del Dr. Zorrilla, que demostraban la circulación, la distribución de los nervios, y otras de notable mérito. Se percata que están en completo abandono, lo que habla del descuido en que cayeron desde temprano estas preparaciones.

A fines del siglo XIX aumenta el número de prosectores, quienes libres de la organización de la enseñanza pudieron dedicarse a la labor científica. Así, Carlos Juliet y Raimundo Charlín, Adolfo Acevedo y el mismo Coste, aportan con disecciones de gran calidad, algunas de las cuales estaban presentes en el gabinete o también llamado ya con propiedad "Museo", igualables sólo a las de Lafargue.

\section{El Museo de Anatomía en el Barrio de Independencia}

De la calle San Francisco la Escuela de Medicina junto al museo llegan el año 1889 a la calle "La Cañadilla” como se conoció al antiguo Camino Real del Inca (posteriormente se conocería como Independencia luego de entrar triunfante el ejército libertador en 1818). Ahí ocupaba el primer piso de la izquierda destacando la presencia de esqueletos, la estatua de Auzoux y otros preparados guardados en muebles de madera. Al disector Coste le continuaría Martín Valenzuela quién sería designado en 1889 director del ahora Museo de Anatomía. Al ascender a Jefe de Trabajos prácticos, se designaría disector a don Luis Solís.

Debido al aumento en el número de los alumnos y a los malos olores que desde Anatomía expelían los cadáveres al resto del inmueble, el Dr. Alejandro del Río solicita el traslado de las dependencias de anatomía en enero de $1890^{4}$. En esa fecha, disector de J.J. Aguirre era don Luis Solís, y de Orrego Luco don Adolfo Acevedo.

\section{La llegada del museo a la calle Zañartu:}

En 1918 se incendia la Escuela de Química y Farmacia ubicada en la esquina de las calles Independencia y el Panteón, actual Zañartu, lo que define el traslado de Anatomía a este sector. Gracias a las gestiones del decano Amunátegui y del profesor Vargas Salcedo se obtienen recursos de la Dirección de Obras Públicas, los que alcanzan a los \$ 186.000 aprobándose el 16 de junio de 1920 el reglamento de funcionamiento de Anatomía y su Museo según consta en la memoria del Director de la Escuela de medicina de la época. Se denomina ahora Instituto de Anatomía y queda sometido a la Escuela de Medicina, siendo dirigido por un Inspector del Instituto y del Museo Anatómico, quién debe ser médico titulado de la Universidad de Chile y así cuidar por siempre su patrimonio. Su entrada sería por la calle Profesor Zañartu, en homenaje a uno de los muertos por la dictadura de Ibáñez cambiando a 1.130 su numeración.

Son los profesores quienes organizan y disponen del funcionamiento del Museo. El Inspector debe velar por el orden y aseo del Museo, poner a disposición de los profesores el material docente adecuado, hacer preparaciones anatómicas, adquirir aquellas que no se pudiesen conseguir en el Museo, conseguir obras, generar publicaciones periódicas, instrumentos, formar el Museo Anatómico, etc. Se designa un Comité de Vigilancia, formado por el director de Escuela Dr. Edmundo Jaramillo, el profesor de anatomía Dr. Roberto Aguirre Luco y su jefe de trabajo práctico, el Dr. Gustavo Jirón, quién dirige luego el Instituto de Anatomía, creando los departamentos de diafanización, momificación y corrosión a cargo del especialista Dr. Jurgen Baumann, fomentando la conservación de las piezas del Museo además de crear una sección de anatomía plástica, hasta 
hace 40 años atrás la única en Sudamérica, que permite copiar con fidelidad cuadros anatómicos tomados del cadáver; tallados primero en cera, se reproducen luego en látex o yeso. Funda la anatomía radiológica, impartiéndola en los trabajos prácticos estimulando la investigación, inculcando el más severo respeto al cadáver humano.

\section{La sala de teratología}

A instancias del profesor Jirón Latapiat comienza a formarse en 1922 la colección de fetos malformados con la llegada de un feto de sexo femenino que poseía una sirenomelia de la maternidad de Lebu. Gran importancia tuvo la tesis sobre "anatomía fetal, cavidad abdominopélvica" de la Dra. Erna Mahn en 1933 en que utiliza más de 800 fetos y el trabajo presentado por el Dr. José Torres Ortega y por su hijo el Dr. Arturo Jirón Vargas en las jornadas bianuales de Anatomía celebradas en diciembre de 1956: "Breves consideraciones en la anatomía del feto y del recién nacido"4. Se reciben muestras hasta el año 1958 siendo en su momento el más completo y diverso de América alcanzando a tener más de 300 fetos malformados en su colección. Muchos fueron donados a nuestra universidad y llegaron de todas las maternidades del país, principalmente de los Hospitales del Salvador y José Joaquín Aguirre, según nos señalararía en el año 2012 Fortunato Gavilán, antiguo funcionario encargado del Museo. Estos fetos se solicitaron para intentar explicar el aumento en la cantidad de malformaciones observadas y así buscar relaciones entre malformaciones y distintos productos químicos, entre los que se destaca la exposición al uso de fertilizantes e insecticidas en madres que trabajaban en agricultura, así como medicamentos como la talidomida en la década de los 50. Sometidos a autopsias primero, luego fueron estudiados con radiografías, tomografías y reconstrucciones tridimensionales.

\section{La sala de Cuadros}

Llamada así coloquialmente esta sala tiene 475 láminas de ilustraciones anatómicas que se guardaban bajo los asientos del anfiteatro anatómico en una colección que empieza a gestarse el año 1903. Estaban agrupadas en 13 áreas temáticas y fueron realizadas por alumnos, docentes y artistas bajo distintas técnicas como óleo, acuarelas, gráfitos, tinta china, etc. ${ }^{7}$.

El 2 de diciembre de 1943 a las 05 AM. según consta en el diario El Mercurio de la época, se produciría el incendio de la Escuela de Medicina, destruyéndose todas las dependencias menos Anatomía y su Museo, por el traslado antes comentado, constituyéndose por ello en las dependencias más antiguas de la actual escuela de medicina.

En forma paulatina se reúnen piezas anatómicas sin formar un Museo propiamente tal, el que llegó a estructurarse en 1960 gracias al Profesor Humberto Vargas Olmedo, quien dio forma a un Museo de piezas humanas conservadas con diversas técnicas, además de piezas de distinto material como acrílicos, látex, cáñamo, yeso, etc. Fue quién destinó las dependencias respectivas y adecuó su distribución.

Posteriormente, en el año 1988 el Dr. Evaristo Cancino Aguila, director del museo y participante del Primer Coloquio de Museos de la Universidad de Chile realizado en el año 1960, propone que así como al Anfiteatro de Anatomía se le designe con el nombre de José Joaquín Aguirre, al gabinete de anatomía (que venía con piezas de la época de Lafargue) se le denomine Museo de Anatomía Humberto Vargas Olmedo de manera formal, en alusión al profesor que sucede a Gustavo Jirón y que organiza las dependencias y las piezas que conforman este museo, según lo que aparece en la memoria de dicho evento. Ocupa, al parecer, el subterráneo, para posteriormente ser trasladado al segundo piso, al menos la sala de exhibición; son tantas las piezas que se reunen que es necesario disponer de otras dependencias en el subterráneo. Cancino señalaba que era el mejor Museo de la especialidad en Chile y el 20 de Sudamérica y que se incrementaba con piezas confeccionadas por ellos mismos y por aporte de piezas enviadas de las sedes Sur y Oriente de la Facultad de Medicina, existiendo además "un pequeño museo de Teratología único en el mundo con un total de 187 piezas, el cual es de uso restringido para especialistas y no está abierto al público" (Figuras 3 y 4).

El museo anatómico estaba dispuesto en el ala oriente del edificio que ocupa actualmente el Instituto de anatomía y contaba ya con al menos 2.300 piezas divididas según su técnica de conservación:

- Osteología.

- Momificación. 


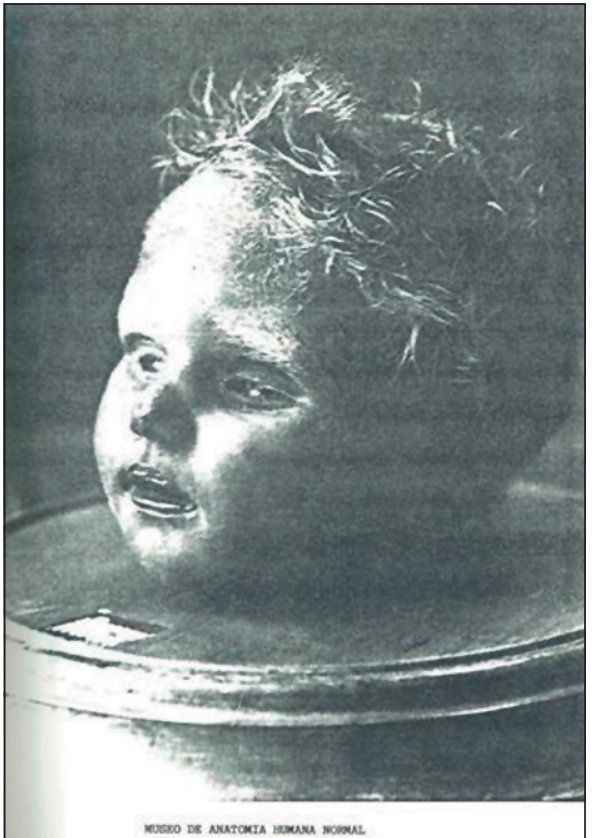

Figura 3. Cabeza de niña, autor: Humberto Fracassi, anatomista argentino. (Fuente ler coloquio museos Universidad de Chile)

- Diafanización.

- Corrosión.

- Parafinados.

- Material Plástico: acrílico, yeso, cartón piedra, cera, goma, cáñamo.

El 99\% de las piezas eran de confección propia y $1 \%$ de obsequios o adquisiciones realizadas en el extranjero. Se disponían en estantes con puertas de vidrio o sobre mesones, disponiendo de una sala de conferencias con capacidad para 30 personas.

Hasta antes de establecerse el programa de donación de cuerpos a fines de los años 70 en que las personas donan su cuerpo voluntariamente a la Universidad de Chile vía poder notarial, los preparados cadavéricos provenían de personas no reclamadas en hospitales y el servicio médico legal, los NN. Por tanto de estos últimos provienen las piezas más antiguas del Museo actual.

Entre las funciones que tenía se encontraban:

- Atender a la facultad de Medicina en su quehacer científico docente de pregrado y postgrado a todas las escuelas de la salud y a otras facultades como Odontología y Bellas Artes, incluso Medicina de la Universidad Católica. Varias

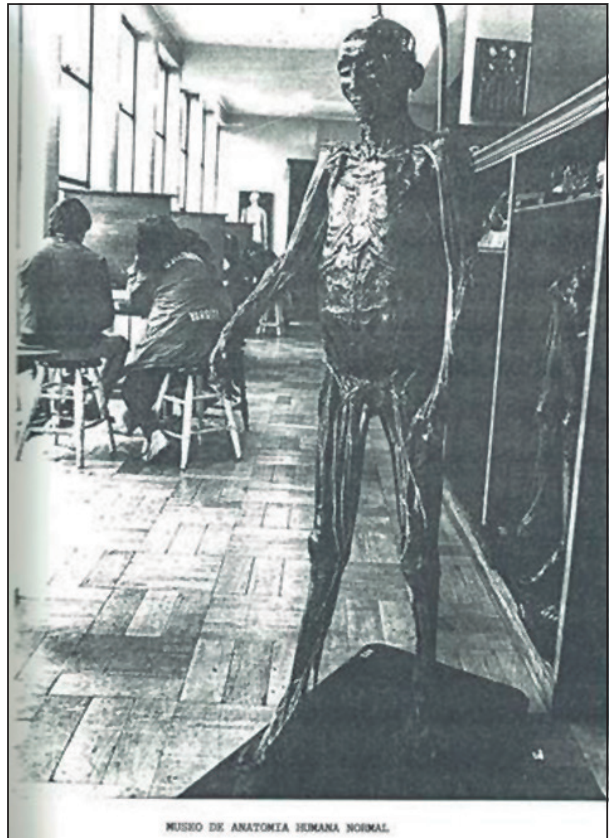

Figura 4. Interior del antiguo museo de calle Zañartu 1.130. (Fuente $1^{\text {er }}$ coloquio museos Universidad de Chile).

piezas de la colección se derivan a las nacientes escuelas de medicina de las Universidades de Concepción (1924) Valparaíso (1950), y Católica (1950) entre otras según antiguos anatomistas.

- El préstamo de piezas anatómicas para montar exposiciones científicas en colegios de enseñanza Media, apoyar sus visitas así como la de Institutos y Fuerzas Armadas.

- Venta de material anatómico para la docencia enviando piezas a diversos centros de enseñanza del país, incluso al extranjero, como España y Puerto Rico. El ingreso era destinado para la mantención y adquisición de nuevo material, pues como sucede hasta hoy en día, el museo no cuenta con presupuesto propio.

Debido a cambios de dirección y de autoridades se pierden estos cargos administrativos, cayendo el museo en un abandono y deterioro evidente, siendo desmantelado el año 2003 con el fin de disponer de espacio para construir nuevas oficinas; la oposición de los estudiantes es fundamental para recuperar la mitad del espacio de la sala de exhibición. 
Se comprende necesario el reposicionar el museo en el resto de la comunidad universitaria generando material informativo sobre su origen y patrimonio, reorganizando las preparaciones en el nuevo espacio recuperado realizando además un proyecto de iluminación con tecnología led. Se gestionan las visitas de instituciones externas, logrando aumentar de 200 a más de 18.000 las visitas al año. Esto le daría un nuevo impulso al Museo, comprendiendo la necesidad de generar investigación en relación a su propia colección, lo que progresivamente conduce a comprender la importancia patrimonial que este lugar tenía no sólo para la Facultad de Medicina, sino también para la Universidad de Chile, y por tanto al país.

Por todo lo anterior en conjunto con la profesora María Paz Valenzuela de la Facultad de Arquitectura, Michele Dinator, tesista de la misma facultad y el Antropólogo Mario Castro, se presentan diversos estudios de distinta índole al Consejo de Monumentos Nacionales el que luego envía un informe al Ministerio de Educación que emite la declaratoria de monumento nacional el día 20 de enero de 2016 del anfiteatro anatómico, sus láminas, el museo y sus colecciones $^{8}$ (Figura 5).

Este es el impulso definitivo para lograr un sitio en la facultad de Medicina, logrando contactos con museos de la Universidad y de otras universidades del país y del extranjero, tomando el desafío de seguir avanzando en su desarrollo museográfico. Se genera una página web del museo: www.museodeanatomia.cl (Figura 6).

Vaya el presente trabajo como agradecimiento y homenaje a todos aquellos quienes cuidaron este patrimonio a través del tiempo.
$\mathrm{N}^{\circ} 612$, de 2015 , de Educación y déjese copia a continuación de éste.

Anótese, tómese razón, regístrese y publíquese.- MICHELLE BACHELET JERIA, Presidenta de la República.- Adriana Delpiano Puelma, Ministra de Educación.

Lo que transcribo a Ud., para su conocimiento.- Valentina Karina Quiroga Canahuate, Subsecretaria de Educación.

(IdDO 988858)

DECLARA MONUMENTO NACIONAL EN LA CATEGORÍA DE MONUMENTO HISTÓRICO AL “ANFITEATRO DEL INSTITUTO DE ANATOMÍA" Y A LAS "COLECCIONES DEL MUSEO DE ANATOMÍA", TODOS PERTENECIENTES A LA UNIVERSIDAD DE CHILE, UBICADOS EN LA COMUNA DE INDEPENDENCIA, PROVINCIA DE SANTIAGO, REGIÓN METROPOLITANA

Núm. 549.- Santiago, 16 de diciembre de 2015.

Considerando:

Que, el Consejo de Monumentos Nacionales, recibió la solicitud de la Consejera Sra. María Paz Valenzuela, representante del Instituto de Historia y Patrimonio de la Universidad de Chile y de los señores, Julio Cárdenas, Director del Museo de Anatomía y Michele Dinator, diseñadora, para declarar como monumento nacional en la categoría de monumento histórico, al "Anfiteatro del Instituto de Anatomía" y a las "Colecciones del Museo de Anatomía", entre las que se consideran las 475 láminas para la enseñanza de la anatomía en la Universidad de Chile, todos pertenecientes a la Universidad de Chile, ubicados en calle Profesor Zañartu $\mathrm{N}^{\circ} 1130$, comuna de Independencia, provincia de Santiago, Región Metropolitana;

Que, los estudios de anatomía en Chile, desde 1823 hasta 1889, se efectuaban en una construcción a los pies del antiguo hospital San Juan de Dios, ubicado en la Alameda a la altura de calle Santa Rosa. En 1889 se inauguró el primer complejo docente para la enseñanza de la medicina en Chile, ubicado en la calle La Cañadilla, en la actual comuna de Independencia;
Figura 5. Extracto Diario Oficial No 41.361, 20 de enero de 2016, cuerpo 1-9. 


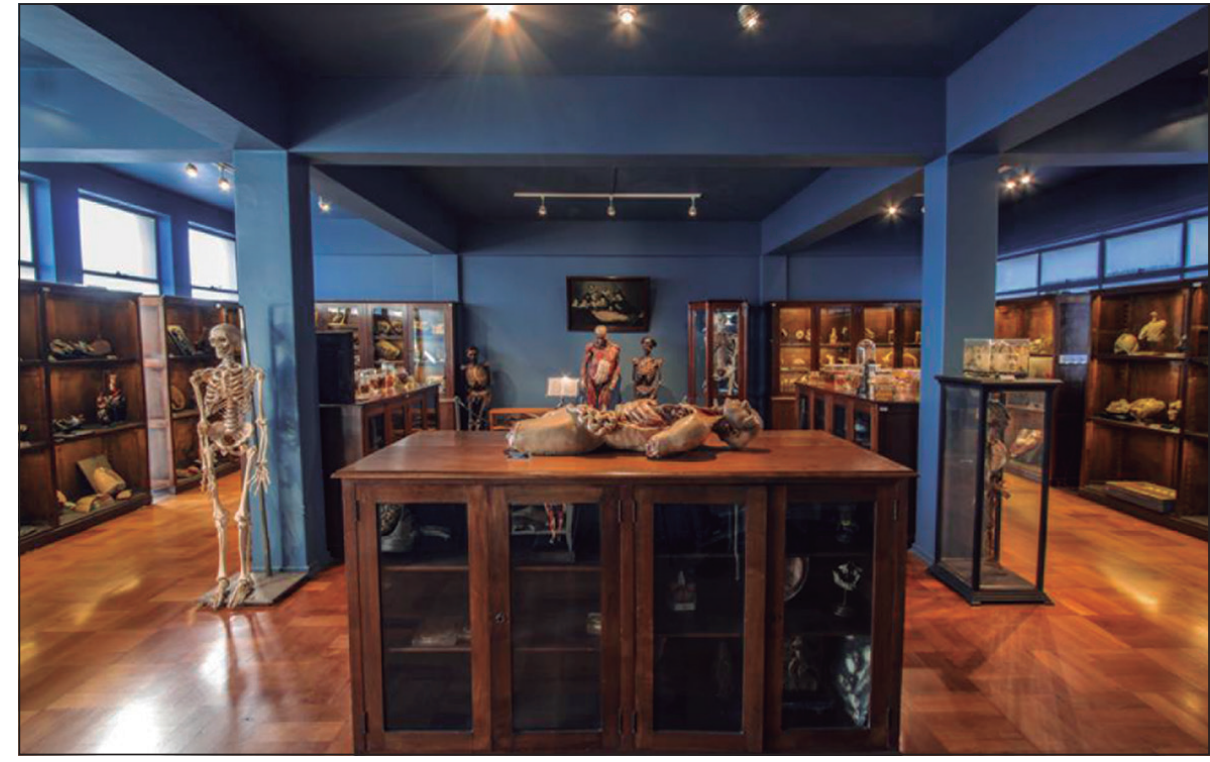

Figura 6. Vista general sala de exhibición museo anatomía. Autor: fotógrafo Marco Saavedra, año 2016.

\section{Referencias}

1. Osorio GC. Historia de la escuela de Medicina de la Cañadilla. Rev Med Chile 2013; 141: 1484-8.

2. Orrego L. Augusto Recuerdos de la Escuela, Revista Médica de Chile, 1922, pág. 5 y sgtes. Santiago de Chile, Imprenta Universitaria.

3. Ferrer PL. Historia General de la medicina en Chile. Imprenta Talca 1904. pág. 353-4.

4. Laval E. Evolución de la Enseñanza de la Anatomía en Chile. Anales Chilenos de Historia de la Medicina. Impreso talleres Servicio Nacional de Salud, 1964, vol 6 (2) pág. 7-75.

5. Boletín de las leyes y de las Órdenes y Decretos del gobierno. Valparaíso, Imprenta del Mercurio, 1846, páginas 357-8, Archivo Biblioteca Nacional. https:// babel.hathitrust.org/cgi/pt?id=mdp.35112103815975;view=1 up;seq=599.

6. Cárdenas VJ. La estatua anatómica del Dr. Auzoux. Primer modelo anatómico de uso docente en Chile. Int J Morphol 2015; 33 (1): 393-9.

7. Cárdenas VJ, Dinator E, Madariaga J, Descripción de las láminas anatómicas de la Universidad de Chile, un monumento nacional. Int J Morphol 2017; 35 (2): 7118.

8. Diario Oficial de la República de Chile. Decreto Número 549, de 2015.- Declara Monumento Nacional en la categoría de Monumento Histórico al "Anfiteatro del Instituto de Anatomía" y a las "Colecciones del Museo de Anatomía", Diario Oficial de la República de Chile, $\mathrm{N}^{\circ} 41.361,20$ de enero de 2016. Cuerpo I-9. http://www.diariooficial. interior.gob.cl/media/2016/01/20/do-20160120.pdf. 